\title{
NP-COMPLETENESS IN THE GOSSIP MONOID
}

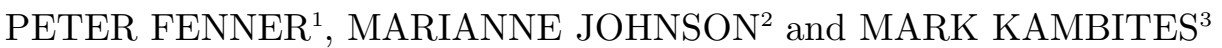 \\ School of Mathematics, University of Manchester, \\ Manchester M13 9PL, England.
}

\begin{abstract}
Gossip monoids form an algebraic model of networks with exclusive, transient connections in which nodes, when they form a connection, exchange all known information. They also arise naturally in pure mathematics, as the monoids generated by the set of all equivalence relations on a given finite set under relational composition. We prove that a number of important decision problems for these monoids (including the membership problem, and hence the problem of deciding whether a given state of knowledge can arise in a network of the kind under consideration) are NP-complete. As well as being of interest in their own right, these results shed light on the apparent difficulty of establishing the cardinalities of the gossip monoids: a problem which has attracted some attention in the last few years.
\end{abstract}

\section{INTRODUCTION}

Gossip problems are concerned with the flow of information through networks with exclusive, transient connections in which nodes, when connected, exchange all known information. The gossip monoid (of rank $n$ ) forms an algebraic model of such a network (with $n$ nodes). It is a semigroup whose elements correspond to possible states of knowledge across the network, and in which the natural multiplication action of a generating set simulates information flow through the establishment of connections.

Gossip monoids are also of considerable interest for purely mathematical reasons: they arise naturally in semigroup theory as the monoids generated by the set of equivalence relations on a finite set, under the operation of relational composition. Since equivalence relations are idempotent, this means gossip monoids are a natural and interesting family of idempotent-generated monoids, the latter being an area of great current interest in semigroup theory (see for example [2, 8, 9, 5, 15].)

Despite the obvious importance of gossip monoids, both within semigroup theory and for applications, remarkably little is known about them. In the 1970s, a number of authors, including Tijdeman [14, Baker and Shostak [1, Hajnal, Milner and Szemeredi 11, independently computed the minimum number of two-way connections required to ensure permeation of all information throughout the network; in semigroup-theoretic terms this is the length of the zero element as a word with respect to a particularly natural set of generators. More recently, Brouwer, Draisma and Frenk [3, 6] have introduced a continuous (tropical) analogue, termed the lossy gossip monoid, which has

\footnotetext{
${ }^{1}$ Email Peter.Fenner@postgrad.manchester.ac.uk. Peter Fenner's research is supported by an EPSRC Doctoral Training Award.

${ }^{2}$ Email Marianne. Johnson@maths . manchester .ac .uk.

${ }^{3}$ Email Mark.Kambites@manchester.ac.uk.
} 
some intriguing connections to composition of metrics and the tropical geometry of the orthogonal group.

Perhaps the most obvious question in this area is: how many elements are there in the gossip monoid of rank $n$ ? In other words, how many distinct knowledge configurations can arise in an $n$-node network of the type discussed above? In 3 this number was calculated computationally for $n \leq 9$, but no obvious pattern emerges from their results, and the hope of extending the sequence much further by similar brute-force computations seems remote. It remains open whether there is an explicit formula for, or even a markedly faster way of counting, the cardinality of the gossip monoid of rank $n$.

In this paper, we consider the complexity of decision problems concerning gossip monoids. Elements of gossip monoids are naturally represented by boolean matrices, and an important problem is to decide, given a boolean matrix, whether it represents a gossip element. One of our main results is that this problem is NP-complete. As well as being of interest in its own right, this sheds some light on the apparent difficulty of determining the cardinality of the gossip monoid, by suggesting that there is no simple combinatorial characterisation of elements, of the kind which might be used to count them. We also establish NP-completeness for a number of other important problems, including Green's $\mathcal{J}$-order.

Besides this introduction, the paper is organised into five sections. Section 2 recalls the definition and basic properties of gossip monoids. Section 3 introduces the algorithmic problems we study, and describes in outline our strategy for establishing their complexity. Sections 4-6 are concerned with the proofs of NP-hardness for these problems.

\section{The Gossip Monoid}

In this section we briefly introduce the gossip monoids and their relationship to knowledge distribution in networks, and discuss some semigroup-theoretic properties of these monoids.

Consider $n$ people ("gossips") each of whom knows a unique piece of information (a "scandal") initially unknown to the others. The people communicate by telephone and in each call the two participants tell each other every scandal they know. The 'gossip problem' (what is the minimum number of calls required before every person knows every scandal?) attracted the attention of a number of researchers in the 1970s, including Tijdeman [14, Baker and Shostak [1, Hajnal, Milner and Szemeredi [11, who proved in a variety of different ways that the minimal number of calls required is:

$$
0 \text { if } n=1, \quad 1 \text { if } n=2, \quad 3 \text { if } n=3, \quad \text { and } 2 n-4 \text { if } n \geq 4 \text {. }
$$

Whilst the gossip problem concerns only the most efficient means to create one particular state of knowledge between the participants, it is clear that understanding the 'gossip state-space' (in other words all possible states of knowledge that can occur in this system) is both a more complicated and more important problem; it is equivalent to understanding the distribution of knowledge within an $n$-node communication network with transient connections, assuming that initially each node possesses a unique piece of information, and that whenever a connection is made nodes exchange all known information. 
We begin by showing that this system can be modelled by considering the right action of a particular monoid (the gossip monoid) on the set of all $n \times n$ boolean matrices, which we use to record these states of knowledge. We write $\mathbb{B}$ for the boolean semiring, that is, the algebraic structure comprising the set $\{0,1\}$ equipped with the operations of maximum (logical "or") and multiplication (logical "and".) Algebraically, this structure is somewhat like a commutative ring, with maximum playing the role of addition, the fundamental difference being that the addition operation is non-invertible. The set $\mathbb{B}_{n}$ consisting of all $n \times n$ boolean matrices (that is, matrices with entries from $\mathbb{B}$ ) forms a monoid under the matrix multiplication induced from the operations in $\mathbb{B}$. (In other words, for $A, B \in \mathbb{B}_{n}$ the $(i, j)$ th entry of the product $A B$ is obtained by taking the maximum over $k$ of the products $a_{i, k} b_{k, j}$. It is easy to see that the 'usual' identity matrix also behaves as an identity with respect to this new multiplication.) Moreover, there is a natural partial order on $\mathbb{B}_{n}$ induced from the natural order on $\mathbb{B}$; for $A, B \in \mathbb{B}_{n}$ we say that $A \preceq B$ if and only if $a_{i, j} \leq b_{i, j}$ for all indices $i, j$. We will then write $A \prec B$ to mean $A \preceq B$ but $A \neq B$.

Each matrix in $\mathbb{B}_{n}$ can be thought of as representing a state of knowledge distribution within an $n$-node network: the entry in row $i$ and column $j$ being 1 exactly if node $j$ (or "person $j$ ", in gossip problem terminology) has learnt the knowledge initially possessed by node $i$ ("scandal $i$ ".) In particular, the identity matrix $I_{n}$ corresponds to the initial state of knowledge (each node knowing only what it knows initially.)

For $i, j \in\{1, \ldots, n\}$ the call matrix $C[i, j]$ is the matrix with 1 s on the main diagonal, and also in the $(i, j)$ and $(j, i)$ positions, and 0 s elsewhere. It is readily verified that if $K \in \mathbb{B}_{n}$ represents a state of knowledge, then the product $K C[i, j]$ represents the state of knowledge resulting by starting in the state represented by $K$ and exchanging all information (a "call") between node $i$ and node $j$. The gossip monoid $G_{n}$ of rank $n$ is the submonoid of $\mathbb{B}_{n}$ generated by the set of all call matrices,

$$
\{C[i, j] \mid i, j \in\{1, \ldots, n\}\} .
$$

Its elements are exactly those matrices modelling states of knowledge which can actually arise in a network of the kind under consideration, starting from the initial state of knowledge and proceeding through calls in which two nodes exchange all information available to them.

In matrix terms, the effect of right multiplication by $C[i, j]$ is to replace columns $i$ and $j$ with their element-wise maximum. Notice in particular that this action is monotonic, in the sense that $K \preceq K C[i, j]$; this corresponds to the fact that nodes do not forget things once learnt. (Dually, one can see that the effect of left multiplication by $C[i, j]$ is to replace rows $i$ and $j$ with their element-wise maximum, and so it is clear that this left action is also monotonic, that is $K \preceq C[i, j] K$.) Notice also that this multiplication respects the partial order, in that $K \preceq L$ implies $K C[i, j] \preceq L C[i, j]$ and $C[i, j] K \preceq C[i, j] L$. Repeated use of these two properties gives us $A C \preceq A B C$ for all $A, B, C \in G_{n}$.

Recall that Green's relations [10, 4] are five equivalence relations $(\mathcal{R}, \mathcal{L}, \mathcal{H}, \mathcal{D}$ and $\mathcal{J})$ and three pre-orders $\left(\leq_{\mathcal{R}}, \leq_{\mathcal{L}}\right.$ and $\left.\leq_{\mathcal{J}}\right)$ which can be defined on any monoid (or semigroup) and play a fundamental role in understanding its structure. In the case of $G_{n}$, the five equivalence relations are easily seen to be trivial — we say that $G_{n}$ is a $\mathcal{J}$-trivial monoid - but the pre-orders remain important and we recall their definitions. 
We define $A \leq_{\mathcal{R}} B$ [respectively, $A \leq_{\mathcal{L}} B$ ] if there exists a $C \in G_{n}$ with $A=B C$ [respectively, $A=C B$ ], and $A \leq \mathcal{J} B$ if there exists $D, E \in G_{n}$ with $A=D B E$. Notice that $A \leq_{\mathcal{R}} B$ if and only if the state of knowledge represented by matrix $A$ can be obtained by starting with the state of knowledge represented by matrix $B$ and applying a sequence of calls.

It is straightforward to verify (as we shall explain below) that the call matrices satisfy the following relations for all distinct values $i, j, k, l$ :

$$
\begin{aligned}
C[i, j] C[i, j] & =C[i, j] \\
C[i, j] C[k, l] & =C[k, l] C[i, j] \\
C[i, j] C[j, k] C[i, j] & =C[j, k] C[i, j] C[j, k] .
\end{aligned}
$$

In terms of gossip, the first set of relations simply record the fact if two people make a repeat call when nothing has occurred in the interim then no information is exchanged in the second call. The second set of relations corresponds to the fact that if the two pairs of callers $\{i, j\}$ and $\{k, l\}$ are disjoint, then it doesn't matter in what order the calls between these pairs take place. (In the situation we are modelling, these communications could in fact occur concurrently.) The third set of relations, which are a kind of braid relation, can easily be verified by matrix multiplication.

It follows immediately from the relations above that $G_{n}$ is the homomorphic image of an infinite 0-Hecke monoid (see [13]) corresponding to the Coxeter presentation with $\left(\begin{array}{l}n \\ 2\end{array}\right)$ involutions $c_{i, j}$ satisfying relations analogous to (2) and (3) above. We also note that the double Catalan monoid $D C_{n}$ studied by Mazorchuk and Steinberg [13] is the submonoid of $G_{n}$ generated by the call matrices $C[i, i+1]$ for $i=1, \ldots, n-1$; in fact the latter can be thought of as an algebraic model of linear networks with exclusive, transient connections in which nodes, when they form a connection, exchange all known information.

The set of all call matrices is a particularly natural set of idempotent generators, which may be generalised as follows. If $S \subseteq\{1, \ldots, n\}$ then the conference call matrix $C[S]$ is the matrix with $1 \mathrm{~s}$ on the main diagonal and in the $(i, j)$ position for all $i, j \in S$, and 0s elsewhere. In terms of knowledge distribution, right multiplication by $C[S]$ models a complete exchange of knowledge between the nodes in $S$; in matrix terms it replaces each column whose index is in $S$ with the element-wise maximum of all columns whose indices are in $S$. (The action of left multiplication by $C[S]$, corresponding to replacing each row whose index is in $S$ with the maximum of all rows whose indices are in $S$, is algebraically dual but less easy to visualise in terms of networks.) It is easy to see that every conference call matrix is an idempotent element of $G_{n}$; indeed it follows from the solution to the original gossip problem [1, 11, 14] that $C[S]$ is a product of 3 call matrices if $|S|=3$ and $2|S|-4$ call matrices if $|S| \geq 4$, these numbers of call matrices being the minimum possible.

We may associate to each element of $\mathbb{B}_{n}$ the binary relation on the set $\{1, \ldots, n\}$ of which it is the adjacency matrix, that is, the relation where $i$ is related to $j$ if and only if the $(i, j)$ entry of the matrix is 1 ; matrix multiplication in $\mathbb{B}_{n}$ then corresponds to relational composition of binary relations. It is easy to see that every call matrix (and indeed every conference call matrix) is the adjacency matrix of an equivalence relation. In fact, equivalence relations correspond exactly to idempotent elements of $G_{n}$ : 
Proposition 2.1. The idempotents in $G_{n}$ are precisely the matrices in $\mathbb{B}_{n}$ which correspond to equivalence relations on $\{1, \ldots, n\}$. (In other words, $A \in G_{n}$ is an idempotent if and only if there exists an equivalence relation $\sim$ on $\{1, \ldots, n\}$ such that $a_{i, j}=1 \Leftrightarrow i \sim j$.)

Proof. First let $A \in G_{n}$ be an idempotent and let $\sim$ denote the binary relation on $\{1, \ldots, n\}$ given by $i \sim j$ if and only if $a_{i, j}=1$. Since $A$ is a product of call matrices, it is clear that $a_{i, i}=1$ and hence $i \sim i$ for all $i$. Since $A$ is idempotent we note that $a_{i, j}=\max _{k}\left\{a_{i, k} a_{k, j}\right\}$, and hence whenever $i \sim k$ and $k \sim j$, we must also have $i \sim j$. To see that $\sim$ is symmetric we note that $A$ can be written as a product of call matrices, $A=A_{1} \cdots A_{m}$ for some $m$, and since $A$ is idempotent we have $A^{m}=A$. We can therefore write $A$ as a product which contains $A_{m}, \ldots, A_{1}$ as a scattered subsequence. Since right multiplication by call matrix $C[i, j]$ replaces columns $i$ and $j$ with their maximum and left multiplication by the same matrix replaces rows $i$ and $j$ with their maximum, we see that $A_{m} \cdots A_{1}=A^{T}$. We therefore have $A^{T} \preceq A$, and so if $i \sim j$ then $j \sim i$.

For the converse, let $\sim$ be an equivalence relation on $\{1, \ldots, n\}$ and let $A$ be the corresponding adjacency matrix. It is easy to see that $A$ is the product (in any order) of the conference call matrices corresponding to the equivalence classes of $\sim$, so that $A \in G_{n}$. It remains to show that $A$ is an idempotent. It follows from reflexivity of $\sim$ that $B:=A^{2} \succeq A$, since $b_{i, j} \geq a_{i, j} a_{j, j}$. Finally we note that if $b_{i, j}=1$, then there exists $k$ such that $a_{i, k} a_{k, j}=1$. By transitivity of $\sim$ we conclude that $a_{i, j}=1$, hence showing that $A^{2}=A$.

Corollary 2.2. $G_{n}$ is the submonoid of $\mathbb{B}_{n}$ generated by the adjacency matrices of equivalence relations.

Proof. One containment follows from the fact that call matrices (which by definition generate $G_{n}$ ) are adjacency matrices of equivalence relations, and the other from the fact that, by Proposition 2.1, $G_{n}$ contains all adjacency matrices of equivalence relations.

Throughout this paper, when the sizes are understood we write 1 for the matrix consisting entirely of ones, 0 for the zero matrix and $I_{n}$ for the identity matrix. Similarly, we shall write $\underline{0}$ to denote the vector of zeros and $\underline{1}$ to denote the vector of all ones.

\section{Algorithmic Problems in the Gossip Monoid}

We shall consider the following decision problems concerning the gossip monoids:

- the Gossip Membership Problem (GMP) is to determine whether a given $A \in \mathbb{B}_{n}$ is a member of $G_{n}$;

- the Gossip $\mathcal{J}$-Order Problem (GJP) is to determine, given matrices $X, Y \in G_{n}$, whether $X \leq_{\mathcal{J}} Y$, that is, whether there exist matrices $U, V \in G_{n}$ with $U Y V=$ $X$

- the Gossip Transformation Problem (GTP) is to determine, given matrices $X, Y \in$ $\mathbb{B}_{n}$, whether there exists a matrix $G \in G_{n}$ such that $X G=Y$;

- the Maximal Gossip Transformation Problem (MGTP) is the restriction of GTP to pairs of matrices $X, Y$ where the matrix $X$ satisfies the maximal column 
condition: $X$ is non-zero and the set of distinct columns of $X$ form an antichain. (In other words, every column is non-zero and maximal among the set of columns.)

Of these problems, the first three are of clear importance in their own right. GMP is critical for understanding the gossip monoid, and also has an obvious application to deciding whether a given knowledge configuration is (absolutely) reachable in a network. GTP corresponds to understanding orbits under the natural action of $G_{n}$ on $\mathbb{B}_{n}$, and hence whether one given knowledge configuration is reachable from another. The $\mathcal{J}$ order is the key to understanding the ideal structure of a $\mathcal{J}$-trivial monoid, and so GJP is essential for understanding the semigroup-theoretic structure of $G_{n}$. The final problem, MGTP, is less natural but is included because it functions as a stepping stone in the proof of NP-hardness for GMP.

By monotonicity, and using the fact that there are only $n(n-1)$ zeros in the identity matrix, we see that there is a polynomial bound on the length of elements of $G_{n}$ as a product of the (quadratically many) call generators. (In [3] it is shown that this polynomial bound can be lowered to $n(n-1) / 2$.) It is therefore possible for a nondeterministic polynomial time computation to guess an element of $G_{n}$. This clearly suffices to show that all of the above problems are in NP.

The remainder of the article is therefore concerned with establishing NP-hardness. We first show that MGTP (and hence also GTP) is NP-hard, by a relatively straightforward reduction from the Dominating Set Problem. Then we show that GJP is NP-hard by a reduction from GTP. Finally, GMP is shown to be NP-hard by a (rather more complex) reduction from MGTP.

We briefly recall the definition of the Dominating Set Problem. Given an undirected graph $H$ with vertex set $V$ and edge set $E \subseteq V \times V$, we say that $D \subseteq V$ is a dominating set for $H$ if every vertex is either in $D$ or adjacent to a vertex in $D$. Given a graph $H$ with vertex set $V$ and a positive integer $k \leq|V|$, the Dominating Set Problem is to decide whether $H$ admits a dominating set of size at most $k$. The problem is known to be NP-complete [7, Problem GT2, Appendix A1] via a transformation from Vertex Cover. (See for example [12, Theorem 2.8.6] for details.)

\section{NP-completeness of the Gossip Transformation Problems}

In this section we show that the Gossip Transformation Problem and the Maximal Gossip Transformation Problem are NP-complete. We show the NP-hardness of these problems with a polynomial time reduction from the Dominating Set Problem.

Theorem 4.1. GTP and MGTP are NP-complete.

Proof. We have already seen that both are in NP, and since MGTP is a restricted version of GTP it suffices to show that MGTP is NP-hard. As discussed above, we do this by reduction from the Dominating Set Problem.

Let $H$ be a graph, say with vertex set $V=\{1, \ldots, n\}$, and let $1 \leq k \leq n$. We will construct matrices $A, B \in \mathbb{B}_{3 n}$, with $A$ satisfying the maximal column condition, such that $A G=B$ for some $G \in G_{3 n}$ if and only if $H$ admits a dominating set of size at most $k$. 
If $k=n$, then $V$ is a dominating set with size at most $k$, so we let $A=B=I_{2 n}$. Otherwise, define a matrix $M \in \mathbb{B}_{n}$ by

$$
m_{i, j}=1 \Longleftrightarrow(i=j) \text { or }(i \text { and } j \text { are adjacent in } H .)
$$

It is easy to see that $D \subseteq\{1, \ldots, n\}$ is a dominating set for $H$ if and only if $\max _{j \in D}\left(m_{i, j}\right)=1$ for every $i$, so there exists a dominating set of size at most $k$ if and only if there exists a set of $k$ or fewer columns of $M$ whose maximum is $\underline{1}$. Clearly, such a set of columns exists if and only if there is a set of $k$ or fewer maximal columns of $M$ whose maximum is $\underline{1}$.

$M$ has at least one maximal column, $m$. Replace each non-maximal column of $M$ with $m$ and call the resulting matrix $M^{\prime}$. This ensures that $M^{\prime}$ satisfies the maximal column condition but no columns are added which are not already columns of $M$. Note that finding the non-maximal column vectors only requires $n(n-1) / 2$ vector comparisons, so $M^{\prime}$ can be computed in polynomial time. Let

$$
\left.A=\left[\begin{array}{c:c:c}
\overbrace{}^{\prime} & 0 & 0 \\
0 & 1 & 1 \\
\hdashline 0 & 0 & 0
\end{array}\right]\right\} n
$$

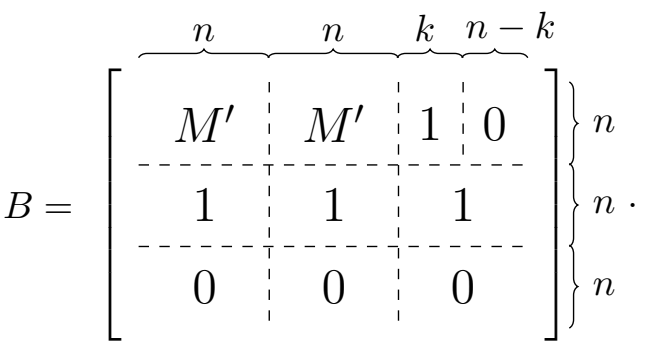

Note that the matrix $A$ satisfies the maximal column condition since $M^{\prime}$ does, and $A$ and $B$ give an instance of the problem $M G T P$.

If $H$ admits a dominating set of size $k$ or less then there is a set of $k$ or fewer columns of $M^{\prime}$ whose maximum is $\underline{1}$. We can multiply matrix $A$ on the right by call matrices which copy each of these columns into a unique column between $2 n+1$ and $2 n+k$. These call matrices will not affect the first $n$ rows of columns $1, \ldots, n$ but will put a 1 in each of the first $n$ rows in columns $2 n+1, \ldots, 2 n+k$. Multiplying this product on the right by the conference call matrix $C[\{2 n+1, \ldots, 2 n+k\}]$ and by each of the call matrices $C[i, n+i]$ for $1 \leq i \leq n$, in any order, will therefore result in matrix $B$. The product of these call matrices is thus an element $G \in G_{3 n}$ such that $A G=B$.

Conversely, if there is a $G \in G_{3 n}$ such that $A G=B$, then there is a sequence of call matrices whose product is $G$, say $G=C_{1} C_{2} \cdots C_{q}$. Without loss of generality we may clearly assume that $A C_{1} \cdots C_{p} \prec A C_{1} \cdots C_{p} C_{p+1}$ for all $p$.

Since right multiplication by a call matrix has the effect of replacing two columns with their maximum, it is clear that each column of each product $A C_{1} \cdots C_{p}$ must be equal to the maximum of some collection of columns of $A$. In particular, the first $n$ rows of each column must equal the maximum of some collection of columns of $M^{\prime}$. In the product $B=A C_{1} \cdots C_{q}$ the first $n$ rows of column $n+i$, for $1 \leq i \leq n$, equal the $i$ th column of $M^{\prime}$. By monotonicity and the fact that $M^{\prime}$ satisfies the maximal column condition, the first $n$ rows of column $n+i$ must equal either 0 or the $i$ th column of $M^{\prime}$ for every product $A C_{1} \cdots C_{p}$. 
Claim. For each $r \in\{2 n+1, \ldots, 2 n+k\}$, there is at most one matrix $C_{p}$ of the form $C[i, r]$ such that $i \leq 2 n$ and the two products $A C_{1} \cdots C_{p-1}$ and $A C_{1} \cdots C_{p}$ differ on column $r$.

Proof of claim: Assume for contradiction that the sequence $C_{1}, \ldots, C_{q}$ contains call matrices $C_{s}=C[i, r]$ and $C_{t}=C[j, r]$, in that order, with $i, j \leq 2 n$ and $2 n+1 \leq r \leq$ $2 n+k$, such that the products $A C_{1} \cdots C_{s-1}$ and $A C_{1} \cdots C_{s}$ differ on column $r$ and the products $A C_{1} \cdots C_{t-1}$ and $A C_{1} \cdots C_{t}$ also differ on column $r$.

We observe that the last $2 n$ rows of column $r$ are identical in $A$ and $B$, so by monotonicity the differences in column $r$ occur in the first $n$ rows. Let $v_{s}, v_{t} \in \mathbb{B}^{n}$ be the first $n$ rows of column $r$ in the matrices $A C_{1} \cdots C_{s}$ and $A C_{1} \cdots C_{t}$, respectively. Since the differences occur in the first $n$ rows, and by monotonicity, we have $0 \prec v_{s} \prec v_{t}$. As the last call matrix in the product $A C_{1} \cdots C_{s}$ is $C_{s}=C[i, r]$, columns $i$ and $r$ must be identical in this matrix, and so $v_{s}$ is also equal to the first $n$ rows of column $i$. If $i \leq n$ then $v_{s}$ is equal to column $i$ of $M^{\prime}$. Otherwise $n<i \leq 2 n$ and since $v_{s}$ is non-zero it is equal to the $(i-n)$ th column of $M^{\prime}$. Either way, $v_{s}$ is equal to a column of $M^{\prime}$ and similar observations show that $v_{t}$ is also equal to a column of $M^{\prime}$. Now $v_{s} \prec v_{t}$ contradicts the maximal column condition on $M^{\prime}$, and this proves the claim.

It follows from the claim that right-multiplying $A$ by the product $C_{1} \cdots C_{q}$ copies at most $k$ of the columns of $M^{\prime}$ into the columns $2 n+1, \ldots, 2 n+k$. But since the result of this right multiplication is to place $1 \mathrm{~s}$ in the first $n$ rows of these columns, it must be that 1 is a maximum of at most $k$ columns of $M^{\prime}$. It follows from our observations that $H$ admits a dominating set of size at most $k$.

\section{NP-Completeness of the Gossip $\mathcal{J}$-order Problem}

To show GJP is NP-hard we will give a polynomial time reduction from GTP. This reduction uses the following lemma, which allows us to "nest" an arbitrary boolean matrix inside a gossip matrix with polynomially larger size:

Lemma 5.1. Let $n \geq 2$. For any $A \in \mathbb{B}_{n}$, the following matrix lies in $G_{n(n+1)}$ :

$$
\left.X=\left[\begin{array}{c:c}
\overbrace{1} & A \\
n^{2} & n \\
1 & 1
\end{array}\right]\right\} n^{2}
$$

Proof. We shall show that $X$ can be written as $X=X_{1} X_{2} X_{3} X_{4}$, where the matrices $X_{i}$ are defined in terms of conference call matrices as follows

$$
\begin{array}{ll}
X_{1}=C[\{n+1, \ldots, n(n+1)\}], & X_{4}=C\left[\left\{1, \ldots, n^{2}\right\}\right], \\
X_{2}=\prod_{i=1}^{n} C[\{i+n(j-1): 1 \leq j \leq n\}], & X_{3}=\prod_{j=1}^{n} C\left[\left\{i+n(j-1): a_{i, j}=1\right\} \cup\left\{n^{2}+j\right\}\right],
\end{array}
$$

and where the products taken in $X_{2}$ and $X_{3}$ can be in any order. 
Each matrix in the product $X_{2}$ is of the form $C[\{i+n(j-1): 1 \leq j \leq n\}]$ for some $i$. This matrix has a 1 in the $a, b$ position if and only if either $a=b$, or $a, b \leq n^{2}$ and $a$ and $b$ are both congruent to $i$ modulo $n$. The product $X_{2}$ therefore has a 1 in the $a, b$ position if and only if either $a=b$, or $a, b \leq n^{2}$ and $a$ and $b$ are congruent modulo $n$. The result is that the top-left $n^{2} \times n^{2}$ block of $X_{2}$ consists of an $n \times n$ array of copies of $I_{n}$ :

$$
\left.X_{2}=\left[\begin{array}{c:c:c:c}
I_{n} & \cdots & I_{n} & \\
\hdashline \vdots & \ddots & \vdots & 0 \\
n^{2} & n \\
I_{n} & \cdots & I_{n} & \\
\hdashline 0 & & I_{n}
\end{array}\right]\right\} n
$$

For $1 \leq j \leq n$ let $S_{j}=\left\{i: a_{i, j}=1\right\} \subseteq\{1, \ldots, n\}$ and let $A_{[j]}$ be the matrix which is the same as $A$ on column $j$ but zero elsewhere. For each $j$ we have

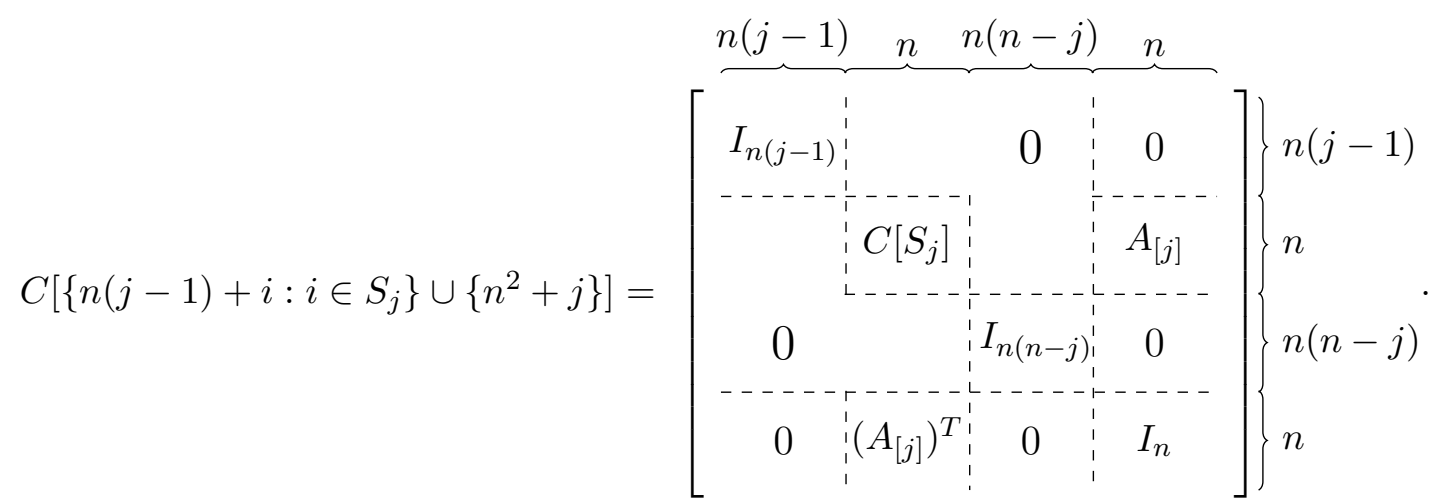

Notice that, as $j$ varies, the corresponding conference call matrices are between disjoint sets of nodes. The product of all such matrices, as $j$ ranges between 1 and $n$, is therefore

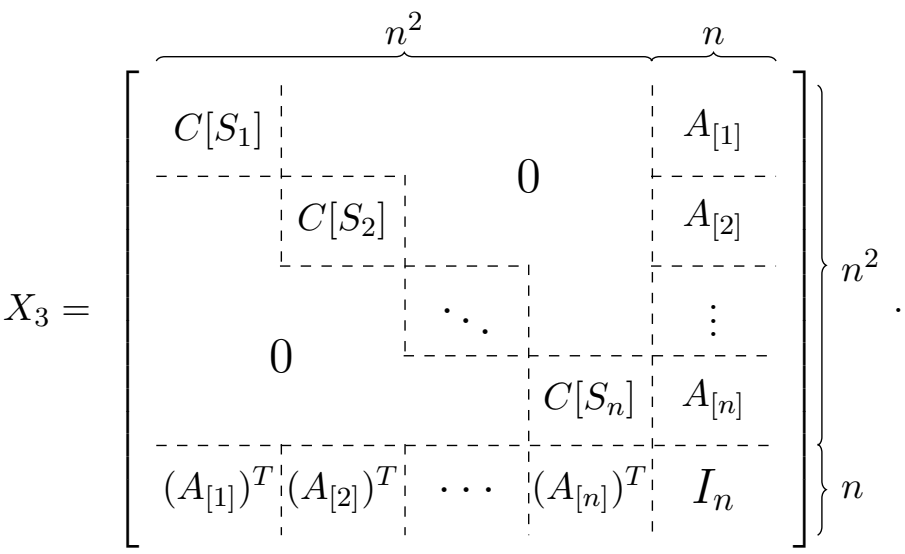


Consider the effect of multiplying $X_{3}$ on the left by $X_{2}$. This leaves the last $n$ rows unchanged. The remaining $n^{2}$ rows can be split into $n$ blocks of $n$ rows each. The structure of the identity matrices in $X_{2}$ means that in $X_{2} X_{3}$ these blocks are all identical to each other and equal to the element-wise maximum of the $n$ corresponding blocks in $X_{3}$. Since the element-wise maximum of $A_{[1]}$ to $A_{[n]}$ is $A$, we get

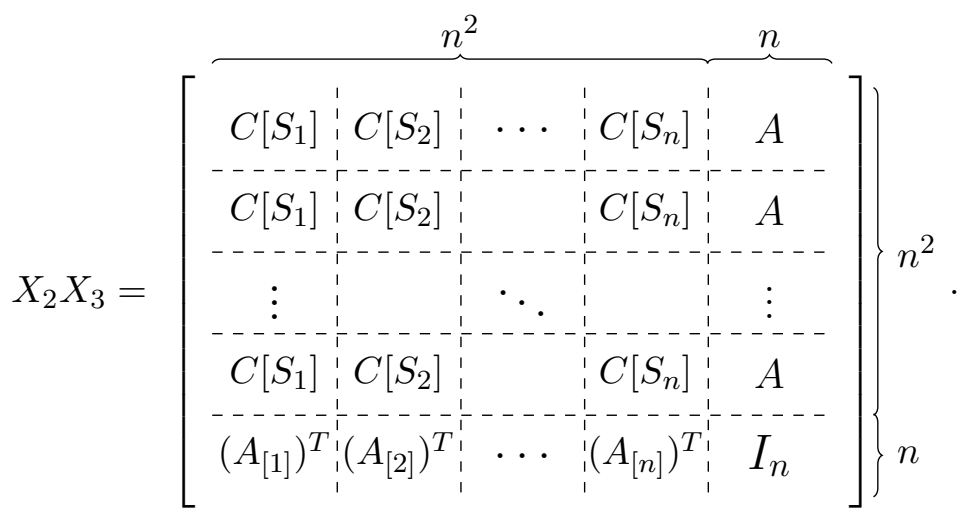

Between them, the last $n^{2}$ rows of $X_{2} X_{3}$ contain a 1 in each column (each conference call has only $1 \mathrm{~s}$ on its diagonal.) Therefore, multiplying on the left by $X_{1}=C[\{n+$ $1, \ldots, n(n+1)\}]$ fills each of these rows with 1 s. Now, between them, the first $n^{2}$ columns of $X_{1} X_{2} X_{3}$ contain a 1 in each row, so multiplying on the right by $X_{4}=C\left[\left\{1, \ldots, n^{2}\right\}\right]$ fills each of these columns with $1 \mathrm{~s}$. We therefore obtain $X_{1} X_{2} X_{3} X_{4}=X$, as required.

Theorem 5.2. GJP is NP-complete.

Proof. We have already seen that the problem is in NP. We show the problem is NP-hard by reduction from MGTP. Given matrices $A, B \in \mathbb{B}_{n}$, let

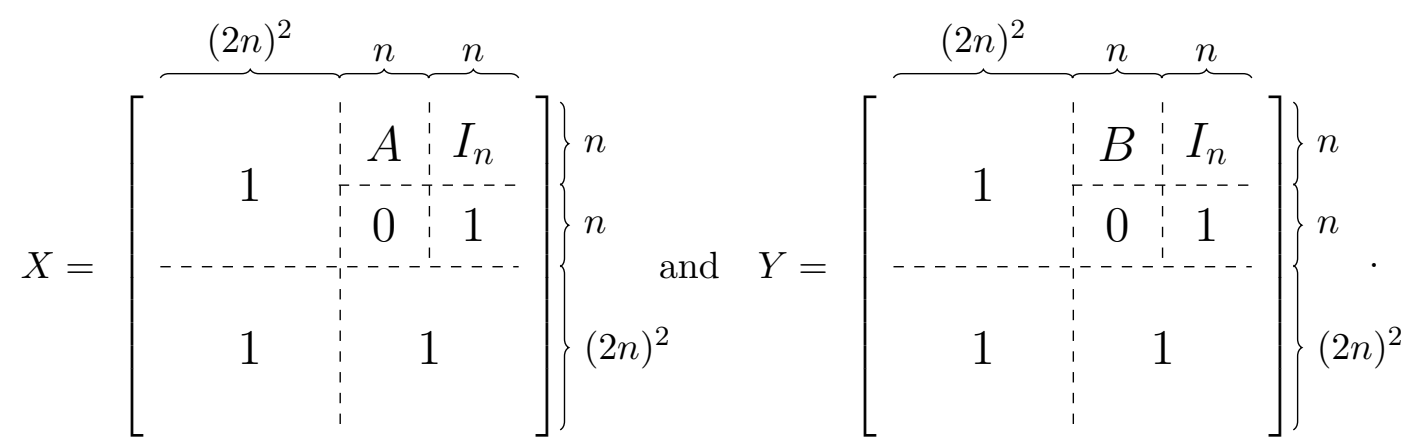

These can clearly be constructed in polynomial time, and by Lemma 5.1 we know that $X, Y \in G_{2 n(2 n+1)}$.

If there exists $G \in G_{n}$ such that $A G=B$ then a simple calculation shows that $U X V=Y$ (and hence $Y \leq_{\mathcal{J}} X$ ) where 


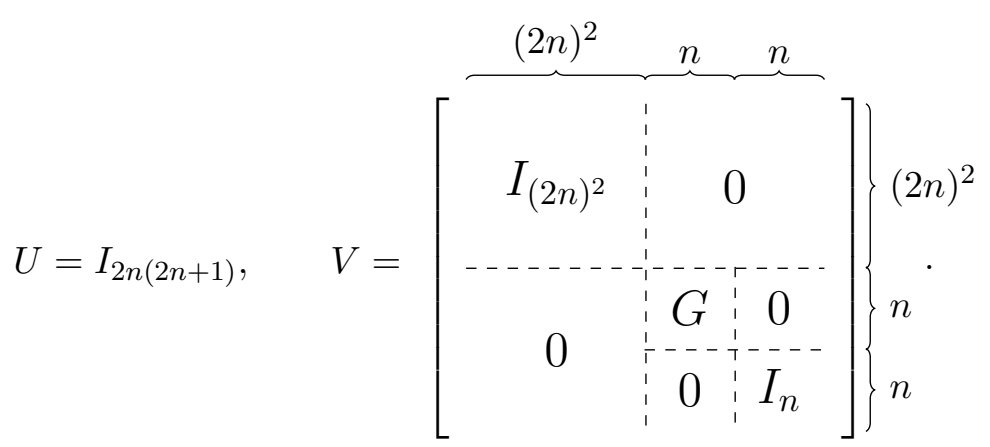

Conversely, if there exist $U, V \in G_{2 n(2 n+1)}$ such that $U X V=Y$, then we consider what structure the matrices $U$ and $V$ could possibly have by considering them as products of call matrices $C[i, j]$.

Recall that left multiplication of $X$ by $C[i, j]$ replaces rows $i$ and $j$ of $X$ with their (element-wise) maximum, whilst right multiplication by $C[i, j]$ replaces columns $i$ and $j$ of $X$ with their maximum. Thus, by regarding $U$ and $V$ as products of call generators, we see that $Y$ can be built from $X$ by successively replacing either two rows or two columns with their maximum. The last $(2 n)^{2}$ rows of $X$ all contain 1s in each of the last $2 n$ columns, whilst the first $2 n$ rows of $Y$ each have a 0 in one of the last $2 n$ columns. It follows that $U$ cannot contain $C[i, j]$ as a factor if $i \leq 2 n$ and $j>2 n$. Each of the rows $n+1, \ldots, 2 n$ of $X$ contain 1 s in each of the last $n$ columns, whilst the first $n$ rows of $Y$ each have a 0 in one of the last $n$ columns. Thus $U$ cannot contain $C[i, j]$ as a factor if $i \leq n$ and $n<j \leq 2 n$. The factors of $U$ are therefore call matrices $C[i, j]$ such that $i$ and $j$ are either both less than $n$, both between $n$ and $2 n$, or both greater than $2 n$. Both $X$ and $Y$ contain the $n \times n$ identity matrix in the top right corner. It follows that $U$ cannot contain $C[i, j]$ as a factor if $i, j \leq n$ are distinct, as this would result in an off-diagonal zero in this $n \times n$ submatrix of $Y$. Therefore, for some $D \in G_{n}, E \in G_{(2 n)^{2}}$ we must have

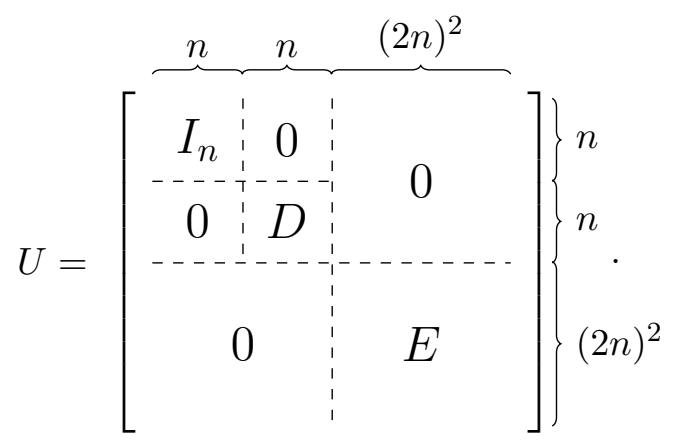

It follows that $X$ and $U X$ are identical in the first $n$ rows. But we have $X \preceq U X \preceq$ $U X Y=Y$ and $X$ and $Y$ are identical in all the other rows, so it must be that $X=U X$, and $X V=U X V=Y$.

Consider now the action of $V$ by right multiplication on $X$. The first $(2 n)^{2}$ columns of $X$ all contain 1s in each of the first $2 n$ rows, whilst the last $2 n$ columns of $Y$ each have a 0 in one of the first $2 n$ rows, and so $V$ cannot contain $C[i, j]$ as a factor if $i \leq(2 n)^{2}$ and 
$j>(2 n)^{2}$. Each of the last $n$ columns of $X$ contains a 1 in row $n+1$, whilst columns $(2 n)^{2}+1, \ldots,(2 n)^{2}+n$ of $Y$ each contain a 0 in row $n+1$, and so $V$ cannot contain $C[i, j]$ as a factor if $(2 n)^{2}<i \leq(2 n)^{2}+n$ and $j>(2 n)^{2}+n$. It now follows that there must be some $F \in G_{(2 n)^{2}}, G, H \in G_{n}$ such that

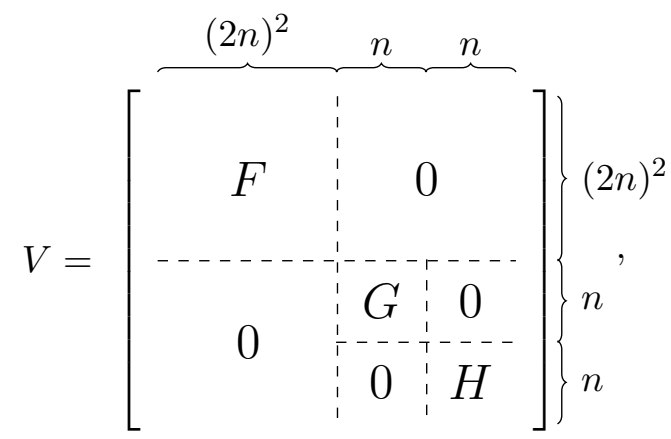

and then $X V=Y$ tells us that $A G=B$ as required.

\section{NP-Completeness of the Gossip Membership Problem}

In this section we prove that the Gossip Membership Problem is NP-complete.

Theorem 6.1. GMP is NP-complete.

Proof. We have already seen that GMP is in NP. We show NP-hardness with a polynomial time reduction from MGTP. Let $A, B \in \mathbb{B}_{n}$ with $A$ satisfying the maximal column condition. Specifically, we show that for each instance $(A, B)$ of the Maximal Gossip Transformation Problem, there exists a Boolean matrix $C \in B_{n(n+4)}$, which can be constructed in polynomial time, for which the problem of deciding membership in the gossip monoid is equivalent to the decision problem MGTP for the pair $(A, B)$. If $n=1$ then we simply set $C=[1]$ if $A=B$ and $C=[0]$ if $A \neq B$. Suppose, then, that $n \geq 2$. Let $C$ be the $n(n+4) \times n(n+4)$ matrix

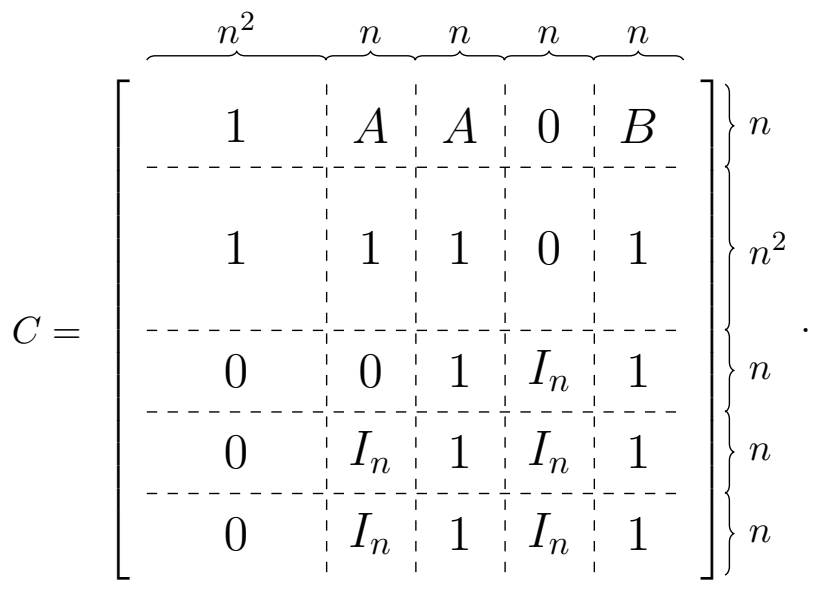

This matrix can clearly be constructed in polynomial time. We claim that $C \in G_{n(n+4)}$ if and only if there is a $G \in G_{n}$ such that $A G=B$. 
For ease of reference during the proof, we shall label the blocks of the matrix (and other matrices of the same size) as follows:

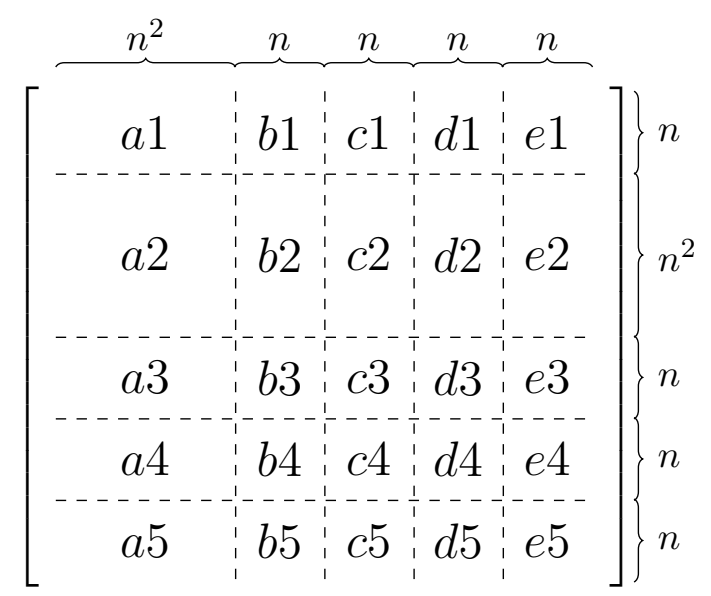

We shall also use $a, b, c, d$ and $e$ to refer to the sets of indices $\left\{1, \ldots, n^{2}\right\},\left\{n^{2}+1, \ldots, n^{2}+\right.$ $n\},\left\{n^{2}+n+1, \ldots, n^{2}+2 n\right\},\left\{n^{2}+2 n+1, \ldots, n^{2}+3 n\right\}$ and $\left\{n^{2}+3 n+1, \ldots, n^{2}+4 n\right\}$ respectively, so that the columns indexed by these sets correspond to the blocks described above. We define $a_{i}=i, b_{i}=n^{2}+i, c_{i}=n^{2}+n+i, d_{i}=n^{2}+2 n+i$ and $e_{i}=n^{2}+3 n+i$ so that, for example, the $i$ th column in block $c$ is column $c_{i}$.

We first show that if $A G=B$ for some $G \in G_{n}$ then $C \in G_{n(n+4)}$, by showing how to write $C$ as a product of call matrices. Let

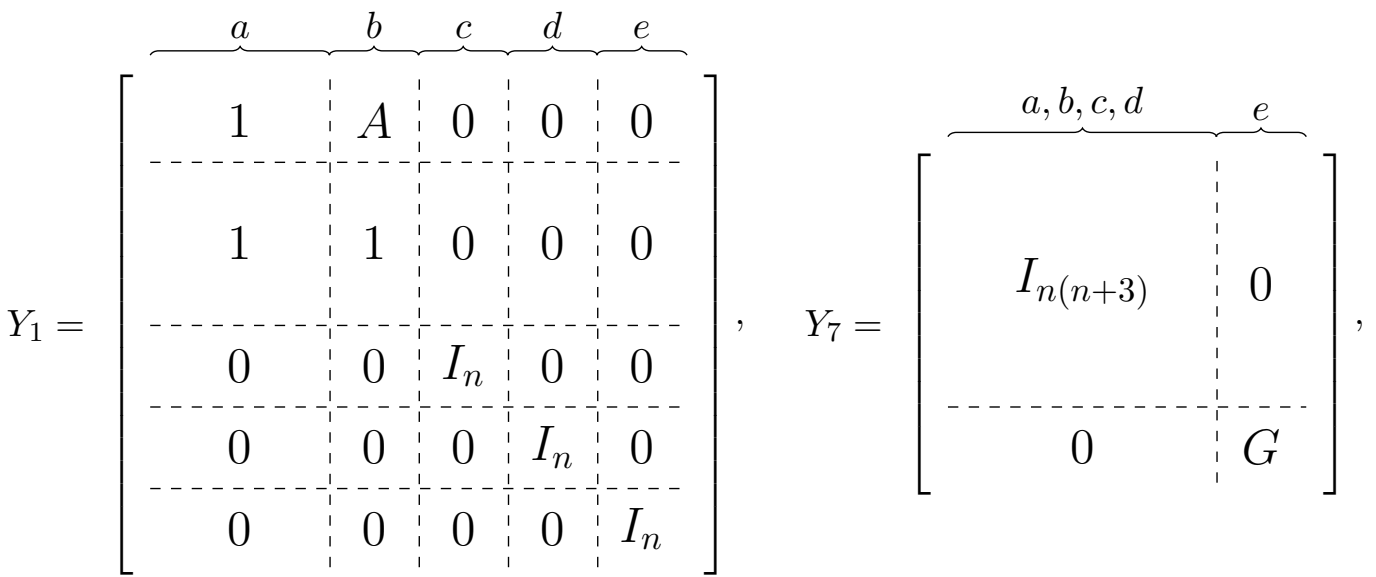

$$
\begin{aligned}
& Y_{2}=\prod_{i=1}^{n} C\left[d_{i}, e_{i}\right], \quad Y_{3}=\prod_{i=1}^{n} C\left[c_{i}, d_{i}\right], \quad Y_{4}=C\left[\left\{c_{1}, \ldots, c_{n}\right\}\right], \\
& Y_{5}=\prod_{i=1}^{n} C\left[b_{i}, e_{i}\right], \quad Y_{6}=\prod_{i=1}^{n} C\left[c_{i}, e_{i}\right] .
\end{aligned}
$$


Since $n \geq 2$ it follows from Lemma 5.1 that $Y_{1}$ is a product of call matrices in $G_{n(n+4)}$. The assumption that $G \in G_{n}$ means that $Y_{7}$ can be written as a product of call matrices in $G_{n(n+4)}$. Matrices $Y_{2}$ through to $Y_{6}$ are all explicitly defined as products of call matrices, and we note that the products in $Y_{2}, Y_{3}, Y_{5}$ and $Y_{6}$ can be taken in any order. It is then straightforward to check that $C=Y_{1} Y_{2} Y_{3} Y_{4} Y_{5} Y_{6} Y_{7}$.

Conversely, assume that $C \in G_{n(n+4)}$ and fix a sequence of call matrices $C_{1}, \ldots, C_{q}$ such that $C=C_{1} \cdots C_{q}$. We may clearly assume without loss of generality that the sequence contains no redundant factors, in the sense that $C_{1} \cdots C_{t} \neq C_{1} \cdots C_{t} C_{t+1}$ (which since multiplication is monotonic means that $C_{1} \cdots C_{t} \prec C_{1} \cdots C_{t} C_{t+1}$ ) for all $t$. By defining a particular scattered subsequence of these factors, we shall construct an element $G \in G_{n}$ satisfying $A G=B$.

We start by observing that for any scattered subsequence $C_{t_{1}}, \ldots, C_{t_{p}}$ of $C_{1}, \ldots, C_{q}$ we must have $C_{t_{1}} \cdots C_{t_{p}} \preceq C$. We proceed by establishing a series of claims.

Claim 1. For each $t$, if $C_{t}$ is of the form $C[i, j]$ with $i \in a \cup b$ and $j \in c \cup d \cup e$, then $C_{t}=C\left[b_{k}, e_{k}\right]$ for some $k \in\{1, \ldots, n\}$. Moreover, for each $k$, the matrix $C\left[b_{k}, e_{k}\right]$ must appear exactly once in the sequence $C_{1}, \ldots, C_{q}$.

Proof of claim: Suppose that $C_{t}=C[i, j]$, where $i \in a \cup b$ and $j \in c \cup d \cup e$. Since $C_{t} \preceq C$ we note that $i \notin a$, otherwise $C$ would contain a 1 in block $a 3, a 4$ or $a 5$. Similarly, we note that $j \notin c \cup d$, since otherwise $C$ would contain a 1 in block $b 3$ or $d 2$. Finally, let $i=b_{k}$ and note that if $j \neq e_{k}$ then $C$ would contain an off-diagonal 1 in block $b 5$.

It now follows that the only way that $C$ can contain the identity matrix in block $b 5$ is for each of the matrices $C\left[b_{k}, e_{k}\right]$ to occur at least once in the sequence $C_{1}, \ldots, C_{q}$. Assume for contradiction that $C\left[b_{k}, e_{k}\right]$ occurs more than once in the sequence, and suppose that $C_{r}$ and $C_{s}$ are the first two occurrences of this matrix. If $C_{t}$ is a factor occurring between $C_{r}$ and $C_{s}$ then $C_{r} C_{t} C_{s} \preceq C$. We cannot have $C_{t}$ of the form $C\left[b_{k}, j\right]$ with $j \in a \cup b$, as otherwise $C_{r} C_{t} C_{s}$ - and therefore also $C$ - would have a 1 in block $a 5$ or an off-diagonal 1 in block $b 5$. Similarly, it cannot be of the form $C\left[e_{k}, j\right]$ with $j \in c \cup d \cup e$, since then $C_{r} C_{t} C_{s}$ would have a 1 in block $b 3$ or $d 2$ or an offdiagonal 1 in block $b 5$. By the first part of the claim, $C_{t}$ cannot be of the form $C\left[b_{k}, j\right]$ with $j \in c \cup d \cup e \backslash\left\{e_{k}\right\}$ or $C\left[e_{k}, j\right]$ with $j \in a \cup b \backslash\left\{b_{k}\right\}$. It also cannot be of the form $C\left[b_{k}, e_{k}\right]$ as it lies strictly between the first two factors of this form. Therefore $C_{t}$ is not of the form $C\left[b_{k}, j\right]$ or $C\left[e_{k}, j\right]$ for any $j$, so $C_{r}$ commutes with $C_{t}$. Since $C_{t}$ was an arbitrary factor between $C_{r}$ and $C_{s}$, and since $C_{r}=C_{s}$ is an idempotent, we have $C_{r} C_{r+1} \cdots C_{s-1} C_{s}=C_{r} C_{s} C_{r+1} \cdots C_{s-1}=C_{r} C_{r+1} \cdots C_{s-1}$, contradicting our assumption that the sequence $C_{1}, \ldots, C_{q}$ contains no redundant factors and thus proving the claim.

For $k \in\{1, \ldots n\}$ let $w_{k}$ be the unique index such that $C_{w_{k}}=C\left[b_{k}, e_{k}\right]$.

Claim 2. For each $k \leq n$, column $k$ of block $e 1$ in the product $C_{1} \cdots C_{w_{k}-1}$ is equal to the zero vector, but the same column in $C_{1} \cdots C_{w_{k}}$ is equal to column $k$ of $A$.

Proof of claim: We first show that in the product $C_{1} \ldots C_{w_{k}-1}$, the $k$ th column of block $e 1$ is equal to the zero vector. Assume that the vector is non-zero, so there is some $C_{s}$ with $s<w_{k}$ such that $C_{1} \cdots C_{s-1}$ has the zero vector in this location, but $C_{1} \cdots C_{s}$ does not. The factor $C_{s}$ must be of the form $C\left[e_{k}, j\right]$ and by Claim 1 and the fact that 
$s<w_{k}$, we have $j \in c \cup d \cup e$. We note that $j \notin c$, otherwise $C_{s} C_{w_{k}}$ - and hence also $C$ - would have a 1 in block $b 3$. Similarly we note that $j \notin e$, otherwise $C_{s} C_{w_{k}}$ would have an off-diagonal 1 in block $b 5$. This means $C_{s}$ must be of the form $C\left[e_{k}, d_{i}\right]$, but then columns $e_{k}$ and $d_{i}$ of $C_{1} \cdots C_{s}$ are identical to each other, so this product has a 1 in block $d 1$, contradicting $C_{1} \cdots C_{s} \preceq C$. Thus the $k$ th column of block $e 1$ in the product $C_{1} \cdots C_{w_{k}-1}$ is equal to the zero vector.

Since the last element of the product $C_{1} \cdots C_{w_{k}}$ is $C_{w_{k}}=C\left[b_{k}, e_{k}\right]$, columns $b_{k}$ and $e_{k}$ of the product are identical and the second part of the claim will follow if the $k$ th column of block $b 1$ in this product is equal to the $k$ th column of $A$. Suppose this does not hold; since it does hold in $C$, the sequence $C_{w_{k}+1}, \ldots, C_{q}$ must include a subsequence which transforms the $k$ th column of $b 1$ into the $k$ th column of $A$. Let $C\left[b_{k}, j\right]$ be one such call. Notice that $j \notin a$, since otherwise $C_{w_{k}} C\left[b_{k}, j\right] \preceq C$ would have a 1 in block a5. Similarly, $j \notin b$, since otherwise $C_{w_{k}} C\left[b_{k}, j\right]$ would have an off-diagonal 1 in block b5. The first part of Claim 1 now shows that the only remaining possibility is $j=e_{k}$. However, this would result in two factors of $C$ both equal to $C\left[b_{k}, e_{k}\right]$, contradicting the second part of Claim 1 . Hence column $k$ of block $b 1$ in the product $C_{1} \cdots C_{w_{k}}$ is equal to the $k$ th column of $A$, completing the proof of the claim.

To provide a convenient starting point for induction, let $C_{0}$ be the $\left(n^{2}+4 n\right) \times\left(n^{2}+4 n\right)$ identity matrix, so that $C=C_{0} C_{1} \cdots C_{q}$.

Claim 3. For all $0 \leq t \leq q$, every column in block $c 1, d 1$ or $e 1$ of $C_{0} C_{1} \cdots C_{t}$ is equal to either $\underline{0}$ or the maximum of some collection of columns of $A$.

Proof of claim: We prove this by induction on $t$. When $t=0$ this is clearly true, as each of these columns is equal to $\underline{0}$. For $t \geq 1$ we shall assume that the condition holds for the columns of the appropriate blocks in $C_{1} \cdots C_{t-1}$. By Claim 1, $C_{t}$ is of the form (i) $C[i, j]$ with $i, j \in a \cup b$, (ii) $C[i, j]$ with $i, j \in c \cup d \cup e$ or (iii) $C\left[b_{k}, e_{k}\right]$ for some $k$. In case (i) the columns of blocks $c 1, d 1$ and $e 1$ are the same as the corresponding columns in the product $C_{1} \cdots C_{t-1}$, which satisfy the condition by assumption. In case (ii) two of the columns of blocks $c 1, d 1$ and $e 1$ are equal to the maximum of the corresponding columns from the product $C_{1} \cdots C_{t-1}$, and so they satisfy the condition of the claim, and the rest of the columns are the same as the corresponding columns from $C_{1} \cdots C_{t-1}$. In case (iii) by Claim 2, column $k$ of block $e 1$ is equal to the $k$ th column of $A$, so satisfies the claim, and all other columns of blocks $c 1, d 1$ and $e 1$ are equal to the corresponding columns in $C_{1} \cdots C_{t-1}$. The claim follows by induction.

To prove the theorem we want to construct a matrix $G \in G_{n}$ such that $A G=B$. Consider those call matrices $C_{t}$ (in order) for which there exists $k \leq n$ such that $w_{k}<t$ and column $k$ of block $e 1$ in the product $C_{1} \cdots C_{t}$ is strictly larger than the same column in the product $C_{1} \cdots C_{t-1}$. (Intuitively, these are the factors which modify some column $k$ of block $e 1$ subsequent to the factor $C_{w_{k}}=C\left[b_{k}, e_{k}\right]$ which copies column $k$ of $A$ into this column.) Let $C_{t_{1}}, \ldots, C_{t_{p}}$ denote the subsequence of these matrices. We shall assume that this sequence is non-empty, since otherwise $B=A$ and $A G=B$ is trivially satisfied by $G=I_{n}$.

Claim 4. Each element of the sequence $C_{t_{1}}, \ldots, C_{t_{p}}$ is of the form $C_{t_{r}}=C\left[e_{j}, e_{k}\right]$ for some $j, k \leq n$ such that $w_{j}<t_{r}$ and $w_{k}<t_{r}$. 
Proof of claim: Let $C_{t_{r}}$ be an element of this subsequence. From the definition of the subsequence there is some $k \leq n$ such that $w_{k}<t_{r}$ and column $k$ of block $e 1$ of $C_{1} \cdots C_{t_{r}}$ is strictly larger than the same column in $C_{1} \cdots C_{t_{r}-1}$. Thus $C_{t_{r}}$ must be of the form $C\left[i, e_{k}\right]$ for some $i \leq n^{2}+4 n$. Claim 1 tells us that $i \notin a$. Since $t_{r}>w_{k}$, Claim 1 also tells us that $i \notin b$. By monotonicity, all columns in block $d 1$ of $C_{1} \cdots C_{t_{r}}$ are $\underline{0}$, so $i \notin d$. Assume for contradiction that $C_{t_{r}}=C\left[c_{j}, e_{k}\right]$ for some $j$.

We observe several relations between columns of various matrices:

- Column $j$ of $A$ is equal to column $j$ of block $c 1$ in $C$.

- By monotonicity, column $j$ of block $c 1$ in $C$ is greater than or equal to the same column in $C_{1} \cdots C_{t_{r}}$.

- Since the last factor in the product $C_{1} \cdots C_{t_{r}}$ is $C_{t_{r}}=C\left[c_{j}, e_{k}\right]$, columns $c_{j}$ and $e_{k}$ are equal in the resulting matrix.

- By our choice of $k$, column $k$ of block $e 1$ of $C_{1} \cdots C_{t_{r}}$ is strictly greater than the same column in $C_{1} \cdots C_{t_{r}-1}$.

- Since $w_{k}<t_{r}$, by Claim 2 and monotonicity we know that column $k$ of block $e 1$ in $C_{1} \cdots C_{t_{r}-1}$ is greater than or equal to column $k$ of $A$.

Putting these together, we find that column $j$ of $A$ is strictly greater than column $k$ of $A$, which contradicts the maximal column condition. Thus $C_{t_{r}}$ must be of the form $C\left[e_{j}, e_{k}\right]$ for some $j$.

We know that $w_{k}<t_{r}$ by definition of the sequence, so for the second part of the claim it is enough to observe that $t_{r} \neq w_{j}$ since $C_{t_{r}} \neq C\left[b_{j}, e_{k}\right]$, and if $t_{r}<w_{j}$ then we would have $C\left[e_{j}, e_{k}\right] C\left[b_{j}, e_{j}\right] \preceq C$ and so $C$ would contain an off-diagonal 1 in block $b 5$, which is not the case.

We see from this claim that there are only two types of call matrix in the sequence which can modify a column of block $e 1$. The first matrix in the sequence which modifies column $i$ of block $e 1$ is $C_{w_{i}}$, and all subsequent matrices (if any) which modify this column are elements of the subsequence $C_{t_{1}}, \ldots, C_{t_{p}}$.

We now define a new sequence of call matrices in $G_{n}$. For $1 \leq r \leq p$, if $C_{t_{r}}=C\left[e_{i}, e_{j}\right]$ then let $D_{r}=C[i, j] \in G_{n}$.

Claim 5. For each $r \leq p$ and $k \leq n$, column $k$ of block $e 1$ of $C_{1} \cdots C_{t_{r}}$ is equal to the zero vector if $t_{r}<w_{k}$, or equal to column $k$ of $A D_{1} \cdots D_{r}$ otherwise.

Proof of claim: By Claim 2, for each $k \leq n$ the $k$ th column of block $e 1$ in the product $C_{1} \cdots C_{w_{k}-1}$ is equal to the zero vector. By monotonicity, if $t_{r}<w_{k}$ then the same column is equal to the zero vector in the product $C_{1} \cdots C_{t_{r}}$. We prove by induction on $r$ that for each $k$ such that $w_{k}<t_{r}$, column $k$ of block $e 1$ of $C_{1} \cdots C_{t_{r}}$ is equal to column $k$ of $A D_{1} \cdots D_{r}$.

Let $t_{0}=0$. Then $t_{0}<w_{k}$ for all $k$ so the claim holds for $r=0$. Now assume the claim holds for $r-1$. That is, for each $k$ such that $w_{k}<t_{(r-1)}$, column $k$ of block $e 1$ of $C_{1} \cdots C_{t_{(r-1)}}$ is equal to column $k$ of $A D_{1} \cdots D_{r-1}$. Given $k \leq n$ such that $w_{k}<t_{r}$ we first consider the differences between column $k$ of block $e 1$ in $C_{1} \cdots C_{t_{(r-1)}}$ and the same column in $C_{1} \cdots C_{t_{r}-1}$. No elements of the subsequence $C_{t_{1}}, \ldots, C_{t_{p}}$ occur among the matrices $C_{t_{(r-1)}+1}, \ldots, C_{t_{r}-1}$ and we have noted that the only other factor of $C$ which can possibly modify column $k$ of block $e 1$ is $C_{w_{k}}$. Thus the two columns under consideration are identical unless $t_{(r-1)}<w_{k}<t_{r}$, and in this case, by Claim 2, column 
$k$ of block $e 1$ in $C_{1} \cdots C_{t_{r}-1}$ is equal to column $k$ of $A$. If $t_{(r-1)}<w_{k}<t_{r}$ then, since $C_{w_{k}}$ comes earlier in the sequence than any other matrix which can modify column $k$ of block $e 1$, we see that $C_{t_{r}}$ is the first element of the sequence $C_{t_{1}}, \ldots, C_{t_{p}}$ which can possibly modify column $k$ of block $e 1$, and thus $D_{r}$ is the first element of the sequence $D_{1}, \ldots, D_{p}$ which can possibly modify column $k$. Thus column $k$ of $A D_{1} \cdots D_{r-1}$ is equal to column $k$ of $A$, and is therefore equal to column $k$ of $e 1$ in $C_{1} \cdots C_{t_{r}-1}$. If $t_{(r-1)}<w_{k}<t_{r}$ does not hold then, since we are only considering $k$ such that $w_{k}<t_{r}$, we must have $w_{k}<t_{(r-1)}$. Thus by the inductive hypothesis we know that column $k$ of block $e 1$ of $C_{1} \cdots C_{t_{(r-1)}}$ is equal to column $k$ of $A D_{1} \cdots D_{r-1}$. Since none of the matrices $C_{t_{(r-1)}+1}, \ldots, C_{t_{r}-1}$ modify this column, column $k$ of $e 1$ in $C_{1} \cdots C_{t_{r}-1}$ is again equal to column $k$ of $A D_{1} \cdots D_{r-1}$. Thus for all $k$ such that $w_{k}<t_{r}$ we know that column $k$ of $e 1$ in $C_{1} \cdots C_{t_{r}-1}$ is equal to column $k$ of $A D_{1} \cdots D_{r-1}$.

By Claim $4, C_{t_{r}}=C\left[e_{i}, e_{j}\right]$ for some $i, j \leq n$ such that $w_{i}, w_{j}<t_{r}$, and then $D_{r}$ is defined to be $C[i, j]$. Let $k<n$ be such that $w_{k}<t_{r}$. We first consider the case when $k \notin\{i, j\}$. Since $k \notin\{i, j\}$, column $k$ of block $e 1$ of $C_{1} \cdots C_{t_{r}}$ is equal to the same column in $C_{1} \cdots C_{t_{r}-1}$. By the previous paragraph, this column is equal to column $k$ of $A D_{1} \cdots D_{r-1}$, and again because $k \notin\{i, j\}$, this is equal to column $k$ of $A D_{1} \cdots D_{r}$. In the case where $k \in\{i, j\}$, column $k$ of block $e 1$ of $C_{1} \cdots C_{t_{r}}$ is equal to the maximum of columns $i$ and $j$ of block $e 1$ of $C_{1} \cdots C_{t_{r}-1}$. Since $w_{i}, w_{j}<t_{r}$, by the previous paragraph this is equal to the maximum of columns $i$ and $j$ of $A D_{1} \cdots D_{r-1}$. Since $D_{r}=C[i, j]$, column $k$ of $A D_{1} \cdots D_{r}$ is also equal to the maximum of columns $i$ and $j$ of $A D_{1} \cdots D_{r-1}$, which completes the proof of the claim.

We now define $G=D_{1} \cdots D_{p}$. For each $k \leq n$, the only factors of $C$ which can possibly modify column $k$ of block $e 1$ are $C_{w_{k}}$ and elements of the subsequence $C_{t_{1}}, \ldots, C_{t_{p}}$. Therefore column $k$ of block $e 1$ of $C$ is equal to the same column in $C_{1} \cdots C_{t_{p}}$ if $w_{k} \leq t_{p}$, or it is equal to the same column in $C_{1} \cdots C_{w_{k}}$ if $t_{p}<w_{k}$.

If $w_{k} \leq t_{p}$ then by Claim 5 column $k$ of $e 1$ in $C_{1} \cdots C_{t_{p}}$ is equal to column $k$ of $A G=A D_{1} \cdots D_{p}$, and therefore column $k$ of $e 1$ in $C$ is also equal to column $k$ of $A G$.

If $t_{p}<w_{k}$ then $C_{w_{k}}$ is the last factor of $C$ to modify column $k$ of block $e 1$, so by Claim 2, column $k$ of $e 1$ in $C$ is equal to column $k$ of $A$. We know from Claim 4 that if $C_{t_{r}}=C\left[e_{j}, e_{k}\right]$ for some $j$ and $r$ then $w_{k}<t_{r}$. However $t_{r} \leq w_{k}$ for all $r \leq p$, so no element of the sequence $C_{t_{1}}, \ldots, C_{t_{p}}$ is equal to $C\left[e_{j}, e_{k}\right]$ for any $j \leq n$. Therefore no element of the sequence $D_{1}, \ldots, D_{p}$ is equal to $C[j, k]$ for any $j \leq n$, and so column $k$ of $A G=A D_{1} \cdots D_{p}$ is also equal to column $k$ of $A$.

In both cases, column $k$ of block $e 1$ of $C$ is equal to column $k$ of $A G$. Therefore $A G$ is equal to block $e 1$ of $C$, which is equal to $B$, and so $G \in G_{n}$ satisfies $A G=B$ as required to complete the proof.

\section{REFERENCES}

[1] B. Baker, R. Shostak, Gossips and telephones. Discrete Mathematics 2, 191-193, 1972.

[2] M. Brittenham, S. W. Margolis, J. Meakin, Subgroups of the free idempotent generated semigroups need not be free, J. Algebra 321 (10), 3026-3042, 2009.

[3] A. E. Brouwer, J. Draisma, B. J. Frenk, Lossy gossip and composition of metrics, Discrete Comput. Geom. 53, 890-913, 2015.

[4] A. H. Clifford, G. B. Preston, The algebraic theory of semigroups. Vol. I., Mathematical Surveys, No. 7 American Mathematical Society, Providence, R.I., 1961. 
[5] I. Dolinka, R. Gray, Maximal subgroups of free idempotent generated semigroups over the full linear monoid, Trans. Amer. Math. Soc. 366 (1), 419-455, 2014.

[6] B. Frenk, Tropical Varieties, Maps and Gossip, PhD. thesis, Eindhoven University of Technology, 2013.

[7] M. R. Garey, D. S. Johnson, Computers and Intractability: A Guide to NP-Completeness. W.H. Freeman and Company, San Francisco, 1979.

[8] R. Gray, N. Ruškuc, On maximal subgroups of free idempotent generated semigroups, Israel J. Math. 189, 147-176, 2012.

[9] R. Gray, N. Ruškuc, Maximal subgroups of free idempotent-generated semigroups over the full transformation monoid, Proc. Lond. Math. Soc. 104 (5), 997-1018, 2012.

[10] J. A. Green, On the structure of semigroups, Ann. of Math. 54, 163-172, 1951.

[11] A. Hajnal, E. C. Milner and E. Szemeredi, A cure for the telephone disease. Canad. Math. Bull. 15, 447-450, 1972.

[12] D. Jüngnickel, Graphs, Networks and Algorithms. Algorithms and Computation in Mathematics, 5. Springer, Heidelberg, 2013.

[13] V. Mazorchuk, B. Steinberg, Double Catalan monoids, J. Algebraic Combin. 36 (3), 333-354, 2012.

[14] R. Tijdeman, On a telephone problem. Nieuw Arch. Wisk. 19 (3), 188-192, 1971.

[15] D. Yang, I. Dolinka, V. Gould, Free idempotent generated semigroups and endomorphism monoids of free G-acts, J. Algebra 429, 133-176, 2015. 